\title{
Immune checkpoint inhibitor combination therapy for gastric cancer: Research progress (Review)
}

\author{
XIAOXU SONG ${ }^{*}$, WEIWEI QI*, JING GUO, LIBIN SUN, AIPING DING, \\ GUANGHUI ZHAO, HUI LI, WENSHENG QIU* and JING LV* \\ Department of Oncology, The Affiliated Hospital of Qingdao University, Qingdao, Shandong 266000, P.R. China
}

Received February 17, 2020; Accepted June 25, 2020

DOI: 10.3892/ol.2020.11905

\begin{abstract}
Gastric cancer is one of the most common types of cancer; notably, gastric cancer is one of the top five malignancies with regards to incidence and mortality rates. The symptoms of early gastric cancer are not typical, exhibiting only slight upper abdominal discomfort. When the symptoms become more obvious, the lesion has usually progressed to an advanced stage. Notably, $>90 \%$ of inpatients already have locally advanced or metastatic gastric cancer at the time of initial diagnosis, with limited treatment options for advanced gastric cancer. These options include chemotherapy, targeted therapy and immune checkpoint inhibitors (ICIs). With regards to ICIs, the clinical benefit of monotherapy for advanced gastric cancer is limited; however, combinations of ICIs and other therapies may have clinical benefit. Relevant clinical studies have demonstrated that combinations of ICIs with chemotherapy, anti-vascular targeted therapy or other molecular targeted therapies, and the use of two ICIs, improve outcomes for patients with advanced gastric cancer. This article is a review of progress in the use of ICIs in combination with other therapies for the treatment of gastric cancer. The purpose of this article was to advance gastric cancer immunotherapy
\end{abstract}

Correspondence to: Professor Wensheng Qiu or Dr Jing Lv, Department of Oncology, The Affiliated Hospital of Qingdao University, 7 Jiaxing Road, Qingdao, Shandong 266000, P.R. China

E-mail: wsqiuqd@163.com

E-mail: lvjing922@126.com

*Contributed equally

Abbreviations: ICI, immune checkpoint inhibitor; HER-2, Human epidermal growth factor receptor-2; VEGF, vascular endothelial growth factor; PD-L1, programmed death ligand 1; PD-1, programmed death protein 1; CTLA-4, cytotoxic T lymphocyte-associated protein 4; ORR, objective response rate; PFS, progression-free survival; OS, overall survival; RFS, recurrence-free survival; DCR, disease control rate; ASCO, American Society of Clinical Oncology

Key words: cancer, gastric cancer, immunotherapy, combination therapy, ICIs, research progress and to improve the overall therapeutic benefit for patients with advanced gastric cancer.

\section{Contents}

1. Introduction

2. ICIs and chemotherapy

3. Anti-CTLA-4 antibody and anti-PD-1/PD-L1 antibody

4. ICIs and anti-vascular targeted therapy

5. ICIs and anti-HER-2 targeted therapy

6. ICIs and other molecular targeted therapies

7. ICIs and other treatments

8. Conclusion and perspectives

\section{Introduction}

Gastric cancer is one of the most common malignancies, which seriously endangers patient health. In 2018, there were 1,033,701 new cases and 782,685 deaths due to gastric cancer worldwide (1). The symptoms of early gastric cancer are minor and usually not easily detected; thus, $>90 \%$ of inpatients with gastric cancer already have locally advanced or metastatic gastric cancer at the time of initial diagnosis, displaying a poor prognosis (2). Treating advanced gastric cancer is difficult, which is the main reason underlying the high mortality rate for gastric cancer. At present, the first-line treatment for advanced gastric cancer is chemotherapy based on platinum drugs and 5-fluorouracil (5-Fu) (3). In addition, trastuzumab has been approved for first-line treatment of patients with human epidermal growth factor receptor-2 (HER-2)-positive gastric cancer (4). The vascular endothelial growth factor (VEGF)-targeted drug, ramucirumab, has also been approved for patients with advanced gastric cancer, for whom first-line treatment protocols have failed. Although there are numerous treatment options for gastric cancer, the overall survival rate for gastric cancer is only $\sim 20 \%$ worldwide $(5,6)$.

With increased understanding of the tumor microenvironment and immune targets, immune checkpoint inhibitors (ICIs) have gradually become a novel treatment method. Immune checkpoint molecules include programmed death protein 1 (PD-1)/programmed death ligand 1 (PD-L1) and cytotoxic T lymphocyte-associated protein 4 (CTLA-4). These molecules 
negatively regulate $\mathrm{T}$-cell activation, such that elimination of their function enhances the immune response, thereby improving the objective response rate (ORR) of patients with cancer (7). ICIs mainly produce anti-tumor effects by blocking PD-1/PD-L1 or CTLA-4 pathways (7). Various PD-1/PD-L1 pathway inhibitors have been approved by the US Food and Drug Administration (FDA) for the treatment of advanced non-small cell lung cancer, renal carcinoma, melanoma and other malignant tumors (8). PD-1/PD-L1 pathway inhibitors, such as pembrolizumab and nivolumab, have effects against advanced gastric cancer; however, as monotherapies they have poor efficiency (9-11).

To circumvent this low efficiency, combined treatment with ICIs and other treatment methods has been considered for clinical gastric cancer benefit. At present, a number of clinical trials of combined immunotherapy are ongoing or have reached their endpoints. Clinical trials can provide evidence for follow-up clinical application, the purpose of which would be to provide more clinical treatment options and more possibilities for improved overall patient treatment benefit. ICI combined treatment programs aim to solve the problem of limited treatment options for advanced gastric cancer, and clinical trials have been conducted to observe the clinical effectiveness and safety of ICI combined treatment programs. Results of clinical trials have suggested that the combination of ICIs with chemotherapy, anti-vascular targeted therapy or anti-HER-2 targeted therapy, and dual ICIs, may improve clinical treatment efficiency of patients with advanced gastric cancer. Clinical trials combining ICIs and radiotherapy for the treatment of advanced gastric cancer are also ongoing. The aim of this article was to review the latest advances in the use of ICIs in combination therapy for advanced gastric cancer, and to explore outstanding issues regarding such treatments.

\section{ICIs and chemotherapy}

In recent years, it has been found that traditional chemotherapy drugs may have an effect on the regulation of the immune pattern. For example, chemotherapy drugs have been reported to enhance the antigenicity of tumor cells (cyclophosphamide, gemcitabine, platinum and paclitaxel) (12) and to enhance sensitivity of tumor cells to immune effector cells (paclitaxel, cisplatin and doxorubicin) (13). Chemotherapy drugs can affect the immune system with direct effects on cytotoxic lymphocytes (paclitaxel, doxorubicin and cisplatin) and elimination of immunosuppressive cells (paclitaxel, gemcitabine and 5-Fu) (14). On the one hand, traditional chemotherapy drugs can enhance the patients' anti-tumor immune response through the aforementioned mechanisms. On the other hand, ICIs can also increase the sensitivity to chemotherapy by enhancing the anti-tumor immune response; ICIs can further eliminate tumor cells that have become resistant after chemotherapy. Therefore, the combination of ICIs with chemotherapy drugs for anti-tumor therapy may present clinical benefits. The relevant clinical trials are described below.

The KEYNOTE-059 trial (NCT02335411) evaluated the efficacy of pembrolizumab combined with cisplatin and 5-Fu as a first-line treatment for patients with HER-2-negative gastric cancer. Cohort 2 enrolled 25 patients that underwent the pembrolizumab + cisplatin $+5-\mathrm{Fu}$ regimen. The ORR was $60.0 \%$ and median progression-free survival (PFS) was 6.6 months $(15,16)$. Cohort 3 enrolled 31 patients that underwent the single-agent pembrolizumab treatment plan. The ORR was $25.8 \%$ and median PFS was 3.3 months (17). Cohorts 2 and 3 of this trial obtained encouraging results, which indicated the obvious advantages of pembrolizumab combined with chemotherapy in the treatment of advanced gastric cancer (Table I).

The KEYNOTE-062 clinical trial (NCT02494583) is a phase III study of locally advanced, unresectable or metastatic gastric cancer. The patients were randomly divided into three treatment groups: Pembrolizumab (200 mg q3w) (referred to as Group P), pembrolizumab (200 mg q3w) + chemotherapy (referred to as Group $\mathrm{P}+\mathrm{C}$ ), and placebo + chemotherapy (referred to as Group C). The chemotherapy regimen was fluorouracil + cisplatin. Among patients with a combined positive score $\geq 1$, the median overall survival (OS) of the three groups was $10.6,12.5$ and 11.1 months, respectively, and the median PFS was 2.0, 6.9 and 6.4 months, respectively. There was a degree of PFS benefit in Group P $+\mathrm{C}$, but the difference in median OS was not statistically significant, thus indicating that the overall benefit for the combined treatment regimen was limited (18).

The ATTRACTION-04 Trial (NCT02746796) is a phase II/III clinical trial, which compared nivolumab + S-1 + oxaliplatin (SOX) and nivolumab + capecitabine + oxaliplatin (CapeOX) as first-line treatments for HER2-negative advanced gastric cancer/gastroesophageal junction cancer. The results revealed that the ORR for the Nivolumab + SOX group was $67 \%$ and was $71 \%$ for the Nivolumab + CapeOX group. The PFS for the Nivolumab + SOX group was 9.9 month, whereas the PFS for the nivolumab + CapeOX group was 7.1 months. An early objective response was observed in $>2 / 3$ of the patients. In addition, $<10 \%$ of patients discontinued treatment due to treatment-related adverse events. There were no treatment-related deaths or new adverse reactions detected in the study (19) (Table I).

At the 2019 American Society of Clinical Oncology (ASCO) annual meeting, there were two studies from China regarding HER2-negative advanced gastric cancer. One was a phase Ib trial of sintilimab combined with a CapeOX regimen as first-line treatment for advanced gastric adenocarcinoma. The results revealed that the ORR was $85 \%$, the disease control rate (DCR) was $100 \%$ and a median PFS was not reached, with no lethal adverse reactions (20). Another was a phase II trial of first-line treatment for advanced gastric adenocarcinoma with camrelizumab and a CapeOX regimen. The results showed that the ORR was $65 \%$, the median PFS was not reached and adverse reactions were controllable (21).

Overall, progress with combinations of ICIs and chemotherapy for advanced gastric cancer has been relatively slow. Results of some clinical trials have not been satisfactory; this is likely due to the gastric cancer tumor cell type and the corresponding tumor microenvironment. Numerous trials of combined ICIs with chemotherapy for advanced gastric cancer are currently underway, including the Checkmate649 trial (NCT02872116; Table I), the KEYNOTE-585 trial (NCT03221426; Table II), and the ATTRACTION-05 trial (NCT03006705) $(22,23)$. Future studies will optimize the treatment sequence, dose intensity and will combine ICIs with 
Table I. Clinical trials of immune checkpoint inhibitor combined therapy for gastric cancer (1st line therapy).

\begin{tabular}{|c|c|c|c|}
\hline Clinical trial & Stage & Combination therapy & Efficacy \\
\hline NCT02335411 Cohort 2 & 2 & $\mathrm{P}+\mathrm{CF}$ & ORR, 60\%; PFS, 6.6 m; OS, $13.8 \mathrm{~m}$ \\
\hline NCT02746796 & $2 / 3$ & $\mathrm{~A}, \mathrm{~N}+\mathrm{SOX} ; \mathrm{B}, \mathrm{N}+\mathrm{CapeOX}$ & A, ORR, 67\%; PFS, 9.9 m; B, ORR, 71\%; PFS, $7.1 \mathrm{~m}$ \\
\hline NCT03382600 & $2 \mathrm{~b}$ & $\begin{array}{l}\mathrm{A}, \mathrm{P}+\text { oxaliplatin }+\mathrm{S}-1 ; \mathrm{B}, \mathrm{P}+ \\
\text { cisplatin }+\mathrm{S}-1\end{array}$ & ORR (ongoing) \\
\hline NCT03409848 & 2 & $\begin{array}{l}\text { A, Trastuzumab + N + I; } \\
\text { B, Trastuzumab + N + mFOLFOX6 }\end{array}$ & OS (ongoing) \\
\hline NCT02872116 & 2 & $\begin{array}{l}\text { A, N + I followed by N; B, CapeOX; } \\
\text { C, FOLFOX; D, N + CapeOX; } \\
\text { E, N + FOLFOX }\end{array}$ & OS (ongoing) \\
\hline NCT03342937 & 2 & $\mathrm{P}+$ Cape OX & PFS (ongoing) \\
\hline
\end{tabular}

OS, overall survival; PFS, progression-free survival; $\mathrm{m}$, months; ORR, objective response rate; P, pembrolizumab; N, nivolumab; I, ipilimumab; $\mathrm{CF}$, cisplatin + 5-fluorouracil; CapeOX, capecitabine + oxaliplatin; FOLFOX/mFOLFOX6, oxaliplatin + 5-fluorouracil + tetrahydrofolic acid

Table II. Clinical trials of immune checkpoint inhibitor combined therapy for gastric cancer (perioperative therapy).

\begin{tabular}{lcll}
\hline Clinical trial & Stage & Combination therapy & Efficacy \\
\hline NCT03221426 & 3 & A, P + XP; B, Placebo + XP; C, P + FLOT; D, Placebo + FLOT & OS and pCR (ongoing) \\
NCT03488667 & 2 & P + mFOLFOX6 before and after surgery & ypRR (ongoing) \\
NCT02918162 & 2 & A, Chemotherapy + P before and after surgery; B, P maintenance & 24 m DFS (ongoing) \\
NCT03257163 & 2 & A, P before surgery; B, P + capecitabine after surgery & RFS (ongoing) \\
\hline
\end{tabular}

OS, overall survival; pCR, pathological complete response; ypRR, response rate after neoadjuvant therapy; DFS, disease-free survival; RFS, recurrence-free survival; $m$, months; P, pembrolizumab; XP, capecitabine + cisplatin; FLOT, docetaxel + oxaliplatin + 5-fluorouracil + tetrahydrofolic acid; mFOLFOX6, oxaliplatin + 5-fluorouracil + tetrahydrofolic acid.

different chemotherapy regimens. Furthermore, it is possible to combine ICIs with chemotherapy, as well as translational therapy or neoadjuvant therapy for gastric cancer. In general, it is necessary to identify the most suitable combination of ICIs with chemotherapy to provide more effective options for clinical treatment of patients of gastric cancer.

\section{Anti-CTLA-4 antibody and anti-PD-1/PD-L1 antibody}

The CTLA-4 and PD-1/PD-L1 signaling pathways can inhibit different aspects of the anti-tumor T-cell response. The CTLA-4 signaling pathway can block the costimulatory signal of T cells, thereby inhibiting the expansion and activation of $\mathrm{T}$ cells in lymph nodes. The combination of PD-1 and PD-L1 can induce T cells to lose their anti-tumor effect, thus producing immune tolerance (24). The combined application of CTLA-4 and PD-1/PD-L1 blocking antibodies has been reported to fully release the killing function of $\mathrm{T}$ cells during the initial and effector stages, avoiding the phenomenon of 'tumor escape' (24). Therefore, the combination of two types of ICIs may result in higher survival benefits for patients with advanced gastric cancer than the use of one ICI alone.

The Checkmate032 trial is an open phase I/II clinical study designed to evaluate the efficacy of the combination of nivolumab (anti-PD-1) and ipilimumab (anti-CTLA-4) for the treatment of advanced solid tumors, including advanced gastric cancer. A total of 160 patients with advanced gastric cancer were randomly divided into three treatment groups: Nivolumab (3 mg/kg) (N3 group), nivolumab (1 mg/kg) + ipilimumab (3 mg/kg) (N1 + I3 group), and nivolumab (3 mg/kg) + ipilimumab (1 mg/kg) (N3 + I1 group). The results revealed that the ORRs of the three groups were 12, 24 and $8 \%$, respectively. Regardless of the tumor PD-L1 expression status, the patients responded to treatment. Patients who were positive for PD-L1 exhibited a higher response rate. The 12-month PFS rates were 8,17 and $10 \%$, respectively. The N1 + I3 group had an ORR of $24 \%$, a DCR of $40 \%$ and an OS of 6.9 months. Hence, the combined treatment of nivolumab $(1 \mathrm{mg} / \mathrm{kg})+$ ipilimumab $(3 \mathrm{mg} / \mathrm{kg})$ had a long-lasting anti-tumor effect for the treatment of advanced gastric cancer, in that the OS of patients was prolonged $(25,26)$. These encouraging results provide evidence for the clinical application of combination therapy and provide new possibilities for clinical treatment of refractory advanced gastric cancer. The phase III study of this clinical trial is currently ongoing, and the results are worth looking forward to.

NCT02340975 is a randomized, multicenter, open-label, phase 1b/2 study, which assessed durvalumab (anti-PD-L1) and tremelimumab (anti-CTLA-4) in combination or as monotherapies for the treatment of chemotherapy-refractory gastric 
cancer. Response rates were low regardless of monotherapy or combination strategies (27). Although the preliminary observations did not detect an obvious benefit of the combined treatment regimen for patients with advanced gastric cancer, the overall safety profiles still provide support for the study of dual ICI combination therapy. This approach could be a new strategy for the treatment of refractory advanced gastric cancer.

In summary, the combination of two ICIs for the treatment of advanced gastric cancer may resolve the problem of low objective efficiency associated with monotherapy. At present, there is little clinical evidence for dual-immune treatment of advanced gastric cancer. Attention needs to be paid to relevant clinical research survival data, in order to determine whether dual-immune treatment of advanced gastric cancer can be applied clinically. In addition, in-depth analysis of the molecular basis for the effect of dual ICIs for the treatment of advanced gastric cancer is essential. In this manner, a molecular basis for clinical application will be evaluated so that new therapeutic targets can be explored.

\section{ICIs and anti-vascular targeted therapy}

In addition to regulating tumor angiogenesis, the vascular endothelial growth factor (VEGF) pathway can regulate the anti-tumor immune response in a variety of manners: i) Preventing the maturation of dendritic cells and effector $\mathrm{T}$ cells, as well as inhibiting the function of cytotoxic $\mathrm{T}$ cells (28); ii) VEGF overexpression promotes the recruitment, differentiation and proliferation of regulatory $\mathrm{T}$ cells (Treg cells) in tumors (29); iii) recruitment of myeloid-derived suppressor cells, which inhibit the anti-tumor response of effector T cells (30); iv) downregulation of endothelial intercellular adhesion molecule-1 in vascular endothelial cells, preventing the migration of effector $\mathrm{T}$ cells into the tumor stroma (28); and v) VEGF-induced tumor blood vessels that produce structural and functional abnormalities, which can increase intra-tumor stress and hinder effector T-cell infiltration (31). The normalization of tumor blood vessels after anti-angiogenesis treatment can reduce intra-tumor pressure, which is beneficial for the infiltration of immune cells into tumor tissues, enhancing the anti-tumor effect of ICIs (32). The aforementioned mechanism provides a theoretical basis for the combined application of anti-vascular targeting drugs and ICIs.

Ramucirumab. Ramucirumab is a fully human monoclonal antibody that binds to the extracellular domain of VEGF receptor and blocks VEGF-mediated tumor neovascularization. The FDA approved ramucirumab for the treatment of patients with advanced gastric cancer and adenocarcinoma of the gastroesophageal junction that have progressed after previous 5-Fu or platinum therapy. For the second-line treatment of advanced gastric cancer, ramucirumab monotherapy or ramucirumab combined with paclitaxel improved the survival of patients. The ORR for monotherapy was only $2.9 \%(33,34)$. Therefore, a treatment plan for ramucirumab combined with ICIs is theoretically reasonable.

NCT02443324 was a phase Ia/b clinical trial of pembrolizumab combined with ramucirumab for the treatment of advanced solid tumors. The clinical trial included 28 patients with advanced gastric cancer or gastroesophageal junction cancer. For the trial, the ORR was $25 \%$, the PFS was 5.3 months, and $96 \%$ of patients had toxic reactions. No treatment-related deaths occurred (35). NCT02999295 is a phase I/II clinical trial of nivolumab combined with ramucirumab for the treatment of advanced gastric cancer (36). The trial included 46 patients with advanced gastric cancer who had failed first-line chemotherapy. After 8 months of follow-up, $44 \%$ of the patients were still receiving treatment, with a partial response rate of $22 \%$, an ORR of $24.4 \%$ and a DCR of $62.2 \%$. NCT02572687 is a phase Ia/b clinical trial of durvalumab combined with ramucirumab for the treatment of patients with advanced gastric or gastroesophageal junction adenocarcinoma who failed first-line or multi-line treatment. The PRR was $17 \%(5 / 29)$, PFS was 2.6 months and OS was 6.4 months (Table III) (37).

Based on these results, ramucirumab combined with ICIs was more effective than ramucirumab monotherapy for the treatment of advanced gastric cancer, with better safety. However, these results are from phase I/II clinical trials, with a need for more relevant phase III/IV clinical studies to confirm results.

Regorafenib. Regorafenib is a multi-target tyrosine kinase inhibitor, which can comprehensively act on relevant targets of tumor angiogenesis, tumor cell proliferation and the tumor microenvironment, exerting multiple anti-tumor effects. Until now, the results of phase II clinical studies have confirmed the clinical value of regorafenib in the treatment of refractory advanced gastric cancer, with related phase III clinical studies planned (38). At ASCO 2019, the Eastern Japan Cancer Center reported the results of the phase Ib REGONIVO study. This study explored the efficacy of regorafenib combined with nivolumab for end-line treatment of microsatellite-stable (MSS) advanced colorectal cancer and gastric cancer. The study included 25 patients with advanced gastric cancer with a median PFS of 5.8 months following treatment. Among them, 11 patients with MSS advanced gastric cancer were observed to have objective treatment responses. The ORR of the gastric cancer group was 44\% (11/25) (39). The results of this study indicated that in patients with MSS gastric cancer, the combined treatment regimen of regorafenib and nivolumab had good anti-tumor activity and good safety. This combined treatment plan is worthy of further investigation in a larger population to determine optimal dosage, treatment sequence and advantaged groups.

\section{ICIs and anti-HER-2 targeted therapy}

HER-2 is a member of the epidermal growth factor receptor family that contains a transmembrane tyrosine kinase, and is involved in the regulation of cell proliferation, differentiation and apoptosis. HER-2 positve levels are higher in tumor cells than in non-tumor cells, with HER-2 overexpression related to tumor size, invasiveness and metastasis of gastric cancer, which affects the OS rate of patients with gastric cancer (40). A global multi-center study in 2018 demonstrated that HER-2 positive rate was $7.3-20.2 \%$ of patients with gastric cancer (41). 
Table III. Clinical trials of immune checkpoint inhibitor combined therapy for gastric cancer (late line therapy).

\begin{tabular}{lcll}
\hline Clinical trial & Stage & Combination therapy & Efficacy \\
\hline NCT02999295 & $1 / 2$ & Ram + N & PR, 22\%; DCR, 59\% \\
NCT02572687 & $1 \mathrm{a} / \mathrm{b}$ & $\mathrm{Ram}+\mathrm{D}$ & ORR, 17\%; PFS, 2.6 m; OS, $6.4 \mathrm{~m}$ \\
NCT02689284 & 2 & $\mathrm{M}+\mathrm{P}$ & ORR, 16\%; DCR, 54\% \\
NCT03413397 & 2 & $\mathrm{P}+$ lenvatinib & ORR (ongoing) \\
NCT03453164 & $1 / 2$ & $\mathrm{~N}+$ radiotherapy & DCR (ongoing) \\
\hline
\end{tabular}

OS, overall survival; PFS, progression-free survival; ORR, objective response rate; DCR, disease control rate; PR, partial response; $\mathrm{m}$, months; $\mathrm{D}$, durvalumab; M, margetuximab; N, nivolumab; P, pembrolizumab; Ram, ramucirumab.

Trastuzumab is an antibody and the first molecularly targeted drug used to treat advanced gastric cancer. The addition of trastuzumab to chemotherapy regimens is currently the standard first-line treatment for HER-2-positive advanced or recurrent gastric cancer (42). Trastuzumab can produce anti-tumor immune effects through antibody-dependent cellular cytotoxicity, antibody-dependent cellular phagocytosis and complement-dependent cytotoxicity (43). The immunoglobulin G1 backbone of trastuzumab is essential to the immune effects of the drug $(44,45)$. In addition, it has been demonstrated that the expression of PD-L1 is upregulated in tumors resistant to trastuzumab (46). Due to the capacity of trastuzumab to affect an anti-tumor immune response, a synergistic anti-tumor effect may be produced in combination with ICIs. The 2019 ASCO annual meeting announced a single-center phase II clinical study in which patients with HER2-positive advanced gastric cancer received trastuzumab + CapeOX regimen combined with pembrolizumab and achieved a median PFS of 11.3 months with an ORR of $87 \%$. Analysis of circulating tumor DNA revealed that the median PFS of the population with ERBB2 amplification was significantly improved compared with that of the population without amplification (14.8 months vs. 7.9 months) (47).

Margetuximab is a novel anti-HER2 monoclonal antibody. NCT02689284 combined margetuximab and pembrolizumab to treat patients with advanced gastric adenocarcinoma who were HER2- and PD-L1-positive, with trastuzumab treatment failure. Among 57 patients with evaluable lesions, ORR was $16 \%$ and DCR was 54\%. Notably, in patients with tumors that exhibited HER-2 amplification (detected with circulating tumor DNA) and were PD-L1 positive, ORR and DCR reached 57 and $86 \%$, respectively. In terms of safety, an adverse reaction rate of grade 3 and above was $13 \%$. No treatment-related deaths occurred (48).

The aforementioned results suggested that for patients with advanced gastric cancer, with HER-2 amplification and PD-L1-positive tumors, the combination of ICI and anti-HER-2 monoclonal antibody can have high survival benefits. However, to apply this combined treatment regimen to clinical practice, more large-scale, multi-center, phase III clinical studies are required. Future studies should aim to determine a reasonable treatment sequence and dose intensity while obtaining considerable efficiency and safety data.

\section{ICIs and other molecular targeted therapies}

Small molecule tyrosine kinase inhibitors, epigenetic regulators and other types of molecular targeted drugs mainly affect the anti-tumor immune response by regulating the expression of immune molecules.

The protein encoded by Dickkopf-1 (DKK1) is involved in inhibition of the Wnt signaling pathway and maintains the immunosuppressive effects of the tumor microenvironment. Notably, DKK1 has been reported to be highly expressed in various tumor types, including gastric cancer (49). Leap Therapeutics announced that its anti-DKK1 antibody, DKN-01, in combination with Merck's anti-PD-1 antibody, Keytruda ${ }^{\circledR}$ (pembrolizumab), demonstrated improved outcomes in patients with advanced gastroesophageal junction and gastric cancer when the tumors expressed high levels of DKK1 (DKK1-high). DKK1-high patients experienced $>22$ weeks median PFS and 32 weeks OS, with a $50 \%$ ORR and $80 \%$ DCR in 10 evaluable patients. DKK1-low patients experienced $\sim 6$ weeks median PFS and $>17$ weeks OS, with a $20 \%$ DCR in 15 evaluable patients (50).

An mTOR inhibitor has been reported to downregulate the expression of PD-L1 in tumor cells and inhibit the activity of FOXP3 ${ }^{+}$Treg cells (51). Histone deacetylase inhibitors and methylation inhibitors can increase the expression of natural killer cell-activating ligands, major histocompatibility complex class 1 and class II molecules, and proinflammatory cytokines. Furthermore, MEK inhibitors can downregulate the expression of immunosuppressive cytokines (IL-1, IL-6, IL-8, IL-10 and VEGF) in the tumor microenvironment (52). The combination of these molecular targeted drugs with ICIs has the possibility of clinical application in gastric cancer. Development of related clinical research will provide more evidence for the effectiveness of broadening the treatment of advanced gastric cancer.

\section{ICIs and other treatments}

Radiotherapy has immune-regulatory effects, which manifest as activation of tumor antigen-specific $\mathrm{T}$ cells to release immunomodulatory factors, increased lymphocyte infiltration into tumors, remodeling of the tumor immune microenvironment and regulation of the tumor immune phenotype (53-55). In addition, radiotherapy can upregulate PD-L1 expression on the surface of tumor cells (53-55). At present, radiotherapy 
is not routinely used for the treatment of gastric cancer, and the combined application of radiotherapy with ICIs in gastric cancer has not been reported. However, clinical studies are ongoing, including a phase I/II clinical study of nivolumab combined with local radiotherapy for unresectable recurrent gastric cancer (NCT03453164) (Table III) (56).

Recently, results achieved with combined treatment with ICIs for other types of tumors have suggested regimens and immunotherapeutic strategies for gastric cancer. For example, the results of the CMP-001-001 study suggested that patients with advanced melanoma treated with Toll-like receptor 9 (TLR-9) agonists combined with pembrolizumab achieved objective and durable tumor regression with tolerable treatment-related toxicity (57). The oncolytic virus drug T-VEC combined with pembrolizumab was used to treat patients with advanced melanoma, with a tumor response rate of $\leq 62 \%$ (58). TLR-9 induces tumor development by affecting the formation of the tumor microenvironment and is abnormally expressed by gastric cancer cells (59). Oncolytic viruses have a direct oncolytic effect and induce the body to initiate anti-tumor immunity. Previous studies have reported that a variety of oncolytic viruses are significantly effective in treating gastric cancer $(60,61)$. Therefore, the aforementioned two combined immunotherapy regimens for melanoma may be theoretically applicable to gastric cancer.

\section{Conclusion and perspectives}

Gastric cancer is one of the most common malignant tumors of the digestive system. Because of limited treatment options for advanced gastric cancer and the low efficiency of single-agent ICIs, combined treatment with ICIs is an attractive strategy for treatment. By consulting the literature, conference reports and browsing relevant official websites, this review reported on treatments for advanced gastric cancer with ICIs in combination with chemotherapy, radiotherapy, targeted therapies and other treatments. Most results suggested that combined treatment plans have improved clinical effectiveness for advanced gastric cancer, with controllable adverse reactions. In addition, there are related clinical studies in progress or that are in the process of recruiting patients, with results anticipated. In our opinion, the future strategies for the use of combined ICIs for the treatment of gastric cancer should be: i) Consider regulation of the tumor immune microenvironment, and explore rationales for the combination of ICIs and other treatment methods for gastric cancer therapy. ii) Conduct multidisciplinary discussions regarding patients with refractory advanced gastric cancer and encourage patients with refractory advanced gastric cancer to participate in clinical trials related to combined treatment with ICIs. iii) Through relevant clinical trials, explore reasonable treatment sequences and reasonable doses of combined ICIs and other treatments for gastric cancer, and define the advantaged groups, providing theoretical support for subsequent clinical application or guideline development. iv) By assessment of other tumor types, integrate data from various clinical studies, proposing different combined treatment options for gastric cancer, evaluate their feasibility and rationale, and actively and rigorously conduct relevant clinical research. v) Assess possible effective markers for screening of gastric cancer dominant populations, and predictors of therapeutic effects. As oncologists, the concept of holistic patient treatment and management to improve the OS of patients with advanced gastric cancer should be ensured.

\section{Acknowledgements}

Not applicable.

\section{Funding}

The present study was supported by the National Natural Science Foundation of China (grant no. 81602068), the Beijing Xisike Clinical Oncology Research Foundation (grant no. Y-BMS2019-038) and the Wu Jieping Medical Foundation (grant no. 320.6750.19088-29).

\section{Availability of data and materials}

The datasets used and/or analyzed during the present study are available from the corresponding author on reasonable request.

\section{Authors' contributions}

WSQ and JL contributed to the planning and design of the study. APD, GHZ and HL were responsible for the literature search. XXS and WWQ contributed by reading the literature and writing the manuscript. JG and LBS performed revisions of the manuscript. All authors have read and approved the final manuscript.

\section{Ethics approval and consent to participate}

Not applicable.

\section{Patient consent for publication}

Not applicable.

\section{Competing interests}

The authors declare that they have no competing interests.

\section{References}

1. Bray F, Ferlay J, Soerjomataram I, Siegel RL, Torre LA and Jemal A: Global cancer statistics 2018: GLOBOCAN estimates of incidence and mortality worldwide for 36 cancers in 185 countries. CA Cancer J Clin 68: 394-424, 2018.

2. Wagner AD, Syn NL, Moehler M, Grothe W, Yong WP Bee-Choo T, Ho JS and Unverzagt S: Chemotherapy for advanced gastric cancer. Cochrane Database Syst Rev 8: CD004064, 2017.

3. Song Z, Wu Y, Yang J, Yang D and Fang X: Progress in the treatment of advanced gastric cancer. Tumour Biol 39: 1010428317714626, 2017.

4. Shah MA, Xu RH, Bang YJ, Hoff PM, Liu T, Herraez-Baranda LA, Xia F, Garg A, Shing M and Tabernero J: HELOISE: Phase IIIb Randomized multicenter study comparing standard-of-care and higher-dose trastuzumab regimens combined with chemotherapy as first-line therapy in patients with human epidermal growth factor receptor 2-positive metastatic gastric or gastroesophageal junction adenocarcinoma. J Clin Oncol 35: 2558-2567, 2017. 
5. Fuchs CS, Tomasek J, Yong CJ, Dumitru F, Passalacqua R, Goswami C, Safran H, Dos Santos LV, Aprile G, Ferry DR, et al: Ramucirumab monotherapy for previously treated advanced gastric or gastro-oesophageal junction adenocarcinoma (REGARD): An international, randomised, multicentre, placebo-controlled, phase 3 trial. Lancet 383: 31-39, 2014.

6. Yazici O, Sendur MA, Ozdemir N and Aksoy S: Targeted therapies in gastric cancer and future perspectives. World J Gastroenterol 22: 471-489, 2016.

7. Ribas A and Wolchok JD: Cancer immunotherapy using checkpoint blockade. Science 359: 1350-1355, 2018.

8. Myint ZW and Goel G: Role of modern immunotherapy in gastrointestinal malignancies: A review of current clinical progress. J Hematol Oncol 10: 86, 2017.

9. Muro K, Chung HC, Shankaran V, Geva R, Catenacci D, Gupta S, Eder JP, Golan T, Le DT, Burtness B, et al: Pembrolizumab for patients with PD-L1-positive advanced gastric cancer (KEYNOTE-012): A multicenter, open-label, phase 1b trial. Lancet Oncology 17: 717-726, 2016.

10. Kang YK, Boku N, Satoh T, Ryu MH, Chao Y, Kato K, Chung HC, Chen JS, Muro K, Kang WK, et al: Nivolumab in patients with advanced gastric or gastro-oesophageal junction cancer refractory to, or intolerant of, at least two previous chemotherapy regimens (ONO-4538-12, ATTRACTION-2) A randomised, double-blind, placebo-controlled, phase 3 trial. Lancet 390: 2461-2471, 2017.

11. Bang YJ, Cho JY, Kim YH, Kim JW, Di Bartolomeo M, Ajani JA Yamaguchi K, Balogh A, Sanchez T and Moehler M: Efficacy of sequential ipilimumab monotherapy vs best supportive care for unresectable locally advanced/metastatic gastric or gastroesophageal junction cancer. Clin Cancer Res 23: 5671-5678, 2017.

12. Zitvogel L, Galluzzi L, Smyth MJ and Kroemer G: Mechanism of action of conventional and targeted anticancer therapies: Reinstating immunosurveillance. Immunity 39: 74-88, 2013.

13. Ramakrishnan R, Huang C, Cho HI, Lloyd M, Johnson J, Ren X, Altiok S, Sullivan D, Weber J, Celis E and Gabrilovich DI: Autophagy induced by conventional chemotherapy mediates tumor cell sensitivity to immunotherapy. Cancer Res 72 : 5483-5493, 2012.

14. Vacchellie E, Aranda F, Eggermont A, Galon J,Sautes-Fridman C, Cremer I, Zitvogel L, Kroemer G, Galluzzi L, Kroemer G and Galluzzi L: Trial watch: Chemotherapy with immunogenic cell death inducers. Oncoimmunology 3: e27878, 2014.

15. Wang Q, Liu F and Liu L: Prognostic significance of PD-L1 in solid tumor: An updated meta-analysis. Medicine (Baltimore) 96 e6369, 2017.

16. Fuchs CS, Doi T, Jang RW, Muro K, Satoh T, Machado M, Sun W, Jalal SI, Shah MA, Metges JP, et al: Safety and efficacy of pembrolizumab monotherapy in patients with previously treated advanced gastric and gastroesophageal junction cancer: Phase 2 clinical KEYNOTE-059 trial. JAMA Oncol 4: e180013, 2018.

17. Smyth $E$ and Thuss-Patience PC: Immune checkpoint inhibition in gastro-oesophageal cancer. Oncol Res Treat 41: 272-280, 2018.

18. Tabernero J, van Cutsem E, Bang YJ, Fuchs CS, Wyrwicz L, Lee KW, Kudaba I, Garrido M, Chung HC, Castro Salguero HR, et al: Pembrolizumab with or without chemotherapy versus chemotherapy for advanced gastric or gastroesophageal junction (G/GEJ) adenocarcinoma: The phase 3 KEYNOTE-062 study. J Clin Oncol 37 (Suppl 18): LBA4007-LBA4007, 2019.

19. Boku N, Ryu MH, Kato K, Chung HC, Minashi K, Lee KW, Cho H, Kang WK, Komatsu Y, Tsuda M, et al: Safety and efficacy of nivolumab in combination with S-1/capecitabine plus oxaliplatin in patients with previously untreated, unresectable, advanced, or recurrent gastric/gastroesophageal junction cancer: Interim results of a randomized, phase II trial (ATTRACTION-4). Ann Oncol 30: 250-258, 2019

20. Xu N, Shen L, Jiang H, Zheng YL, Qian J, Mao CY, Zhou H and Wang SY: Efficacy and safety of Sintilimab in combination with XELOX in first-line gastric or gastroesophageal junction carcinoma (GC/GEJC). J Clin Oncol 37 (Suppl 15): S4042, 2019.

21. Shen L, Peng Z and Zhang YQ: Camrelizumab combined with Capecitabine and Oxaliplatin followed by Camrelizumab and Apatinib as first-line therapy for advanced or metastatic gastric or gastroesophageal junction cancer: Updated results from a multi-center, open label phase II trial. J Clin Oncol 37 (Suppl 15): S4031, 2019.

22. Stein A, Moehler M, Trojan J, Goekkurt E and Vogel A: Immuno-oncology in GI tumours: Clinical evidence and emerging trials of PD-1/PD-L1 antagonists. Crit Rev Oncol Hematol 130: 13-26, 2018.
23. Bang YJ, Van Cutsem E, Fuchs CS, Ohtsu A, Tabernero J, Ilson DH, Hyung WJ, Strong VE, Goetze TO, Yoshikawa T, et al: KEYNOTE-585: Phase III study of perioperative chemotherapy with or without pembrolizumab for gastric cancer. Future Oncol 15: 943-952, 2019.

24. Wei SC, Duffy CR and Allison JP: Fundamental mechanisms of immune checkpoint blockade therapy. Cancer Discov 8: 1069-1086, 2018

25. Janjigian YY, Bendell J, Calvo E, Kim J, Ascieto PA, Sharma P, Ott PA, Peltola KJ, Jaeger D, Evans J, et al: Check Mate-032: Phase I/II open-label study of safety and activity of nivolumab (nivo) alone or with ipilimumab (ipi) in advanced and metastatic (A/M) gastric cancer (GC). J Clin Oncol 34: 4010, 2016.

26. Le DT, Bendell JC and Calvo E: Safety and activity of nivolumab monotherapy in advanced and metastatic gastric or gastroesophageal junction cancer (GC/GEC): Results from the CheckMate-032 Study. J Clin Oncol 34: 6, 2016.

27. Kelly RJ, Lee J, Bang YJ, Almhanna K, Blum-Murphy M, Catenacci DVT, Chung HC, Wainberg ZA, Gibson MK, Lee KW, et al: Safety and efficacy of durvalumab and tremelimumab alone or in combination in patients with advanced gastric and gastroesophageal junction denocarcinoma. Clin Cancer Res 26: 846-854, 2020

28. Ott PA, Hodi FS and Buchbinder EI: Inhibition of immune checkpoints and vascular endothelial growth factor as combination therapy for metastatic melanoma: An overview of rationale, preclinical evidence, and initial clinical data. Front Oncol 5: 202, 2015.

29. Terme M, Pernot S, Marcheteau E, Sandoval F, Benhamouda N, Colussi O, Dubreuil O, Carpentier AF, Tartour E and Taieb J: VEGFA-VEGFR pathway blockade inhibits tumor-induced regulatory T-cell proliferation in colorectal cancer. Cancer Res 73: 539-549, 2013.

30. Gabrilovich D, Ishida T, Oyama T, Ran S, Kravtsov V, Nadaf S and Carbone DP: Vascular endothelial growth factor inhibits the development of dendritic cells and dramatically affects the differentiation of multiple hematopoietic lineages in vivo. Blood 92: 4150-4166, 1998 .

31. Shrimali RK, Yu Z, Theoret MR, Chinnasamy D, Restifo NP and Rosenberg SA: Antiangiogenic agents can increase lymphocyte infiltration into tumor and enhance the effectiveness of adoptive immunotherapy of cancer. Cancer Res 70: 6171-6180, 2010.

32. Jain RK: Normalization of tumor vasculature: An emerging concept in antiangiogenic therapy. Science 307: 58-62, 2005.

33. Fuchs CS, Tomasek J, Yong CJ, Dumitru F, Passalacqua R, Goswami C, Safran H, Dos Santos LV, Aprile G, Ferry DR, et al: Ramucirumab monotherapy for previously treated advanced gastric or gastro-oesophageal junction adenocarcinoma (REGARD): An international, randomised, multicentre, placebo-controlled, phase 3 trial. Lancet 383: 31-39, 2014.

34. Wilke H, Muro K, Van Cutsem E, Oh SC, Bodoky G, Shimada Y, Hironaka S, Sugimoto N, Lipatov O, Kim TY, et al: Ramucirumab plus paclitaxel versus placebo plus paclitaxel in patients with previously treated advanced gastric or gastro-oesophageal junction adenocarcinoma(RAINBOW): A double-blind, randomised phase 3 trial. Lancet Oncol 15: 1224-1235, 2014.

35. Kamath SD, Kalyan A and Benson AB: Pembrolizumab for the treatment of gastric cancer. Expert Rev Anticancer Ther 18: 1177-1187, 2018.

36. A Phase $1 / 2$ Study of Ramucirumab Plus Nivolumab in Participants with Gastric or GEJ Cancer [EB/OL]. (2018-05-04) [2020-05-08]. https://clinicaltrials.gov.

37. Bang YJ, Golan T, Lin CC, Kang YK, Wainberg ZA, Wasserstrom H, Jin J, Mi G, McNeely S, Laing N, et al: Interim safety and clinical activity in patients (pts) with locally advanced and unresectable or metastatic gastric or gastroesophageal junction $(\mathrm{G} / \mathrm{GEJ})$ adenocarcinoma from a multicohort phase I study of ramucirumab (R) plus durvalumab (D). J Clin Oncol 36: 92 , 2018.

38. Pavlakis N, Sjoquist KM, Martin AJ, Tsobanis E, Yip S, Kang YK, Bang YJ, Alcindor T, O'Callaghan CJ, Burnell MJ, et al: Regorafenib for the treatment of advanced gastric cancer (INTEGRATE): A multinational placebo-controlled phase II trial. J Clin Oncol 34: 2728-2735, 2016.

39. Fukuoka S, Hara H, Takahashi N, Kojima T, Kawazoe A, Asayama M, Yoshii T, Kotani D, Tamura H, Mikamoto Y, et al: Regorafenib plus Nivolumab in patients with advanced gastric (GC) or colorectal cancer (CRC): An open-label, dose-finding, and dose-expansion phase 1b trial (REGONIVO, EPOC1603). J Clin Oncol 37 (Suppl 15): S2522, 2019. 
40. Rüschoff J,Dietel M, Baretton G, Arbogast S, Walch A, Monges G Chenard M, Penault-Llorca F, Nagelmeier I, Schlake W, et al: HER2 diagnostics in gastric cancer-guideline validation and development of standardized immunohistochemical testing. Virchows Arch 457: 299-307, 2010.

41. Kim WH, Gomez-Izquierdo L, Vilardell F, Chu KM, Soucy G, Santos L, Monges G, Viale G, Brito MJ, Osborne S, et al: HER2 Status in Gastric and Gastroesophageal Juntion Cancer: Results of the Large, Multinational HER-EAGLE Study. Appl Immunohistochem Mol Morphol 26: 239-245, 2018.

42. Saeki H, Oki E, Kashiwada T, Arigami T, Makiyama A, Iwatsuki M, Narita Y, Satake H, Matsuda Y, Sonoda H, et al: Re-evaluation of HER2 status in patients with HER2-positive advanced or recurrent gastric cancer refractory to trastuzumab (KSCC1604). Eur J Cancer 105: 41-49, 2018.

43. Corraliza-Gorjon I, Somovilla-Crespo B, Santamaria S, Garcia-Sanz JA and Kremer L: New strategies using antibody combinations to increase cancer treatment effectiveness. Front Immunol 8: 1804, 2017.

44. Bahou C, Love EA, Leonard S, Spears RJ, Maruani A, Armour K, Baker JR and Chudasama V: Disulfide Modified IgG1: An investigation of biophysical profile and clinically relevant $\mathrm{fc}$ interactions. Bioconjug Chem 30: 1048-1054, 2019.

45. Sulea T, Rohani N, Baardsnes J, Corbeil CR, Deprez C, Cepero-Donates Y, Robert A, Schrag JD, Parat M, Duchesne M, et al: Structure-based engineering of $\mathrm{pH}$-dependent antibody binding for selective targeting of solid-tumor microenvironment. MAbs 12: 1682866, 2020.

46. Chaganty BKR, Qiu S, Gest A, Lu Y, Ivan C, Calin GA, Weiner LM and Fan Z: Trastuzumab upregulates PD-L1 as a potential mechanism of trastuzumab resistance through engagement of immune effector cells and stimulation of IFN $\gamma$ secretion. Cancer Lett 430: 47-56, 2018.

47. Janjigian YY, Maron SB, Chatila WK, Millang B, Chavan SS, Alterman C, Chou JF, Segal MF, Simmons MZ, Won E, et al: First-line Pembrolizumab (P), Trastuzumab (T), Capecitabine (C) and Oxaliplatin (O) in HER2-positive metastatic esophagogastric adenocarcinoma. J Clin Oncol 37 (Suppl 4): S4011, 2019.

48. Catenacci DV, Park H, Uronis HE, Kang YK, Lacy J, Enzinger PC, Park SH, Lee KW, Ng MCH, Gold PG, et al: Margetuximab (M) plus pembrolizumab (P) in ERBB2-amplified PD-L1+ gastroesophageal adenocarcinoma (GEA) post trastuzumab (T). J Clin Oncol 36 (15 suppl): S4030, 2018.

49. Zhuang GF, Tan Y, Zeng JT, Zhang JW, Tang J, Zeng SP and Qin X: Expression of serum Dickkopf-1 in gastric cancer patients. Asian Pac J Trop Med 8: 870-872, 2015.

50. Wall JA, Klempner SJ and Arend RC: The anti-DKK1 antibody DKN-01 as an immunomodulatory combination partner for the treatment of cancer. Expert Opin Investig Drugs: May 14, 2020 doi: $10.1080 / 13543784.2020 .1769065$
51. Eil R, Vodnala SK, Clever D, Klebanoff CA, Sukumar M, Pan JH, Palmer DC, Gros A, Tamamoto TN, Patel SJ, et al: Ionic immune suppression within the tumor microenvironment limits T cell effector function. Nature 537: 539-543, 2016.

52. Patel SA and Minn AJ: Combination cancer therapy with immune checkpoint blockade: Mechanisms and strategies. Immunity 48: 417-433, 2018.

53. Bernstein MB, Krishnan S, Hodge JW and Chang JY: Immunotherapy and stereotactic ablative radiotherapy (ISABR): A curative approach? Nat Rev Clin Oncol 13: 516-524, 2016.

54. Tang C, Wang X, Soh H, Seyedin S, Cortez MA, Krishnan S, Massarelli E, Hong D, Naing A, Diab A, et al: Combining radiation and immunotherapy: A new systemic therapy for solid tumors? Cancer Immunol Res 2: 831-838, 2014.

55. Deng L, Liang $\mathrm{H}$, Burnette $\mathrm{B}$, Beckett $\mathrm{M}$, Darga $\mathrm{T}$, Weichselbaum RR and Fu YX: Irradiation and anti-PD-L1 treatment synergistically promote antitumor immunity in mice. J Clin Invest 124: 687-695, 2014

56. Checkpoint Inhibitor and Radiotherapy for Recurrent Gastric Cancer (CIRCUIT) [EB/OL]. (201 8-12-1 8) [2020-05-08]. https://clinicaltrials.gov.

57. Milhem M, Gonzales R, Medina T, Kirkwood JM, Buchbinder E, Mehmi I, Niu JX, Shaheen M, Weight R, Margolin K, et al: Abstract CT144: Intratumoral toll-like receptor 9 (TLR9) agonist, CMP-001, in combination with pembrolizumab can reverse resistance to PD-1 inhibition in a phase Ib trial in subjects with advanced melanoma. Cancer Res 78 (13 Suppl): CT144, 2018.

58. Ribas A, Dummer R, Puzanov I, VanderWalde A, Andtbacka RHI, Michielin O, Olszanski AJ, Malvehy J, Cebon J, Fernandez E, et al: Oncolytic virotherapy promotes intratumoral $T$ cell infiltration and improves anti-PD-1 immunotherapy. Cell 174: 1031-1032, 2018.

59. Wang TR, Peng JC, Qiao YQ, Zhu MM, Zhao D, Shen J and Ran ZH: Helicobacter pylori regulates TLR4 and TLR9 during gastric carcinogenesis. Int J Clin Exp Pathol 7: 6950-6955, 2014.

60. Gujar S, Pol JG, Kim Y, Lee PW and Kroemer G: Antitumor benefits of antiviral immunity: An underappreciated aspect of oncolytic virotherapies. Trends Immunol 39: 209-221, 2018.

61. Yokoda R, Nagalo BM, Arora M, Egan JB, Bogenberger JM, DeLeon TT, Zhou Y, Ahn DH and Borad MJ: Oncolytic virotherapy in upper gastrointestinal tract cancers. Oncolytic Virother 7: 13-24, 2018.

This work is licensed under a Creative Commons Attribution-NonCommercial-NoDerivatives 4.0 International (CC BY-NC-ND 4.0) License. 